Ambient Science, 2016: Vol. 03(Sp1); 01-04 DOI:10.21276/ambi.2016.03.sp1.ga01

\title{
An Analysis on Views of Iranian Women about Incentive Policies on Childbearing Decision-making
}

\section{Masoumeh Hosseinpoor', Mahdi Asadi², Fariba Keshavarz', Saeed Sobhanian ${ }^{3 *}$, Athar Rasekh Jahromi ${ }^{4}$, Fatemeh Moloudi ${ }^{5}$}

'Student Research Committee of Jahrom University of Medical Sciences, Jahrom, Iran

${ }^{2}$ Department of Emergency Medicine, Jahrom University of Medical Sciences, Jahrom, Iran

${ }^{3}$ Department of Community Health, Jahrom University of Medical Sciences, Jahrom, Iran

${ }^{4}$ Department of Gynecology and Obstetrics Jahrom University of Medical Sciences, Jahrom, Iran

${ }^{5}$ Department of periodontology, Assistant professor of Shiraz Dental School, Shiraz, Iran.

Study Area: Jahrom, Fars province, Iran

Coordinate: $28^{\circ} 44^{\prime \prime} \mathrm{N} ; 53^{\circ} 20^{\prime \prime} \mathrm{E}$

Key words: Women's insight, Birth control

\section{Abstract}

Women's insight of childbearing toward incentive policies population has always been remaining underneath. In this descriptive study, we enrolled 662 women between the ages of 15 and 49 years. Survey was done by distributing a questionnaire withı 13 questions on incentive policy and three questions on the number of the child they desire to have and who are the decision maker for childbearing and tend to childbearing if incentive policies apply. Most women were seen to support the incentive policies. Most participants wished to have less than 3 children. Surprisingly, 379 participants (57\%) didn't wish to decide about childbearing and repeated pregnancy if the Incentive policies get applied. There are some elements viz., living place, income rate, provide facilities like free foodstuffs basket which would be essential to increase the childbearing decision-making.

rate among Iran's 75 million people has produced a dramatic change in the cover-up of the population now. Some factors such as proper higher education, availability of the proper health network system in remote areas, economic strength, staying in education longer, delaying marriage are likely to have an indirect effect on fertility rate declination. Today, the sociologists of Iran are getting more concerned about the future of the country's population (Kariman et al., 2014). Keeping in mind about the reduced rate of population, in the year 2012, the government of Iran started an incentive policy as per which, Housekeeping has been considered as a career and in which provision of pension also allocated. In addition, policies like 'Monthly reward children', 'increase the duration of maternity leave', 'Mandatory insurance coverage for treatment of mothers and children', 'A monthly salary of unemployed women with young children', 'Free food cart for pregnant women' altogether have also promoted in a large scale. Although childbearing decision-making is a complex process which is usually influenced by various factors (Erfani, 2013) it seems Iranian families did not take much interest to have more children, influenced by incentive policies. With regard to the fact, the women played a major role in decisionmaking for the childbearing and till date very little effort has been made on women's insight about childbearing 
influenced by incentive policies, we decided to investigate the proper reason behind the failure of such childbearing decision-making policy.

\section{Methodology:}

The study was a descriptive-analytic, using stratified randomized sampling among women between the ages of 15 and 49 years. As per the prevalence for childbearing tendency in reported in the previous studies (Shadi-Talab, 2001), we calculated sample size which is as follows; prevalence $=60 \%$, confidence interval $=95 \%$, relative error margins $=10 \%$, and the base population $=100000$. Cochran formula suggested the minimum sample size requirement was 662 individuals from women.

The current study was conducted from October 2013 to February 2015 in the south of Iran. The survey was done on the basis of a questionnaire. The first section contained socio-demographic questions (Age, Education, Marital status, Occupation, Income), followed by the second set which included 13 questions about incentive policy and trend to fertility.

The participants were asked to answer on the basis of five given choices- i) totally disagree, ii) disagree, iii) no comment iv) agree and v) totally agree. Questionnaire included various categories which were as follows: i) low economic status families lead to less likely to fertility, ii) improving the economic status of the family tend to have more children, iii) having more children lowers the welfare of the family, iv)-having more children prevent parents' educational progress, v) childbearing does not conflict with social activities, vi) concerns about the future of children effect on decisions for pregnancy, vii) Providing free food basket by the government may be more incentives for mothers, viii) Considering the monthly salaries for non-working women and housewives can effect on the desire to pregnancy, ix) Compulsory insurance of the mother and the child is effective to have children, $x$ ) Remove the lack of insurance coverage for the fourth child and more is effective to have more children, xi) Increase the duration of maternity leave for working woman could be effective to have more children, xii) In regard to pension insurance for housewives could be effective to have more children, xiii) Number of children is effect on tendency to have children. Further, they were asked three additional questions about the number of the child which they want to have and regarding the same who are the decision maker for the same and tend to childbearing if incentive policies would apply.

Statistical analysis: Data analyses were done by descriptive statistic method; Mean, sd, CI, frequency and etc. Logistic regression was used to predict, changing insight amount among participant. This is dependent variable in the presence of other independent variables.

\section{Results:}

Mean, sd, range and $95 \% \mathrm{CI}$ o demographic questionnaire have been presented in Table-1. A total of 662 participant in their reproductive age in between 15 -49 years (mean age $=29 \pm 8.75$ years) were enrolled in this descriptive-analytic study. Mean income was revealed $391.190 \pm 780.47$ (IRR). Mean marriage duration was found $9.57 \pm 9.14$ year. Mean number of male child was recorded as $5.6 \pm .81$, whereas

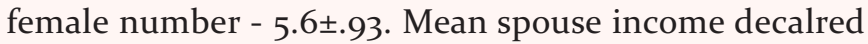
by the participants was $1285 \cdot 79 \pm 870.77$ (Iranian Rial).

The frequency of response with respect to the degree of agreement about incentive policies for childbearing has been showed in Table-2, according to which most of the women were agreeing to accept the incentive policies. One of the important questions was about the number of Childs participants wish to perceive and in response to which most of the participants shown interest in having less than 3 children (Table-3). Due to having some strong traditionally culture/rule, the decision for childbearing depends on some other family members too therefore we asked participant in other question about that and illustrate in Table-4. As it could be seen in the Table-4, $78.3 \%$ of them agree with answer that both woman and man decide about childbearing. The most important question from us was about the repeated pregnancy and child bearing decision making if incentive policies would applied (q16). In response, it is somewhat surprising that $379(57 \%)$ of participants overruled the decision of childbearing and repeated pregnancy. The Logistic regression was used for the prediction of changing insight of participant in the presence of other variables. The goodness of fit test (Hosmer \& Lemeshow Test) with $\mathrm{p}=0.387$ Showed that Logistic regression is possible. The Cox \& Snell R Square and Nagelkerke R Square indicated that variances are explained in this study between 0.120 to o.160. The results of calculation B and $\operatorname{Exp}(B)$ was shown that agreement of childbearing had an inverse correlation with child number and had direct correlation with other variables (Table 5).

Finally the regression Equation:

$$
\begin{aligned}
& \frac{{ }^{*} \mathrm{p}}{1-\mathrm{p}}=\log \left(\text { residency }{ }^{*}\right. \text { o.848-child.no*0.560+q2- } \\
& \left.0.255+\mathrm{q} 7-0.421+\mathrm{q} 12{ }^{*} \mathrm{o} .268+\mathrm{q} 14^{*} \mathrm{o} .822\right) \\
& \text { *: ratio of yes/no in q16 }
\end{aligned}
$$

\section{Discussion:}

During past half century, the reproduction rate in the developing countries decreased but such decrement in Iran was surprising (Abbasi-Shavazi \& McDonald, 2005). It ignites the Iran government to apply such policies which could encourage the Iranian people for bearing more children. Our study was designed to answer few relevant the question- "Do the change in government's policies (social, economic, cultural) cause to change in women's attitude?" According to few earlier survey studies, the 
changes in couple's attitude about childbearing could be manipulated by some big changes in their attitude about reproduction ideals and having children (Abbasi-Shavazi \& McDonald, 2005). Study about the descriptive indicators of samples in this study (table-1), indicated that the samples are reliable for generalizing them to statistical society. Investigating about the redundancy of nearly agreeing and completely agreeing levels in response to the first question, it was found that family ' income is obviously the important factor for increasing the number of children. The current search results revealed that $46.2 \%$ of people declared that due to the ill economic condition they don't want to have more children whereas $39.8 \%$ had stated that improvement in family's economic condition would increase the couple's willingness to have more children. Various studies were about effective elements about couples' attitude and due to which sometimes different results have been reported. For example, the couples in Tabriz are having less income source which bounded them to having more children. It was already reported by Kalantari et al., (2010) that the couples who have less income are more willing to have more children, a somewhat same report was given by Mahmoudi et al. (2013). In Iran women's idea depends on more income (Mahmoudi et al. 2013) nevertheless, Farrokh-Eslamlou et al. (2013) reported that the women's decision is independent of their family income. It may be due to some specif ic religious structure of the society.

Our 2nd set of questionnaire clearly revealed that Iranian women are more willing to have more children as compared to their men and that is only due to their innate willingness. But the process of answering the questions shows that childbearing increscent in society does not only depend on women's willingness as they also get worried about financial hitches. High performance on positive attitude towards agreeing to completely agreeing answers in 3 rd and 4th questions show that most of the participants have a concept that having more children cause to decrease level of welfare and they believe in this subject- "fewer children, More welfare" and which maybe exist in them with regards to their past training. As per Mansourian \& Khoshnevis (2006), proper training is very important in changing ideas/concept and attitude which can influence/alter people's thoughts which are embedded in them since their childhood. However, the approximate equality of agreeing and disagreeing ideas express the existence of a social solution and the importance of social and educational improvement and growth until the existence of the child is not a preventive to this improvement. The study of Aghajanian (1991) supports our result but the Kalantari (2010)'s study do not support our findings. Perhaps, it was because of variation in their existing social structures which are mainly based on their proper education and women's preferment. High frequency of agreeing and completely agreeing ideas in 6th question illustrate that families are always worried about their children's future without any attention to their family's members and this problem is the result of unpredictable and inconsistent economic situation of our country. Farrokh-Eslamlou et al. (2013) reported that in Iran, women worried about the inconsistent economic situation of families. Not so much redundancy of agreeing attitudes in 7 th question show that giving free foodstuffs basket does not make any effect in childbearing decisionmaking. The 8th question shows the positive effects of more income in increasing the childbearing and this subject is confirmed in research elsewhere (Andersson et $a l, 2014)$. The approximate equality of agreeing and disagreeing attitudes in 9th and 1oth question illustrate that about half of the sample society have other worries in addition to compulsory insurance for having children.

Interestingly, the idea of, let the women leave the work even without salary is important to increase women's willingness for having more in families with financial problems. Also, let the women leave the job is more secure for them. But high redundancy of agreeing on the answer in 12th question show that allocates more retirement benef its to housewives that constituted a large group in the society trepan a guarantee for the responsibility that each woman feels to have about her child's future. High controversy regarding agreeing and completely agreeing attitudes on 13th question indicate that in women's concept, the number of existing children in the family is effective on increasing childbearing, but in answer to the question that was about the number of children, most of them want 2 or 3 children. Further, the women also believe that making the decision about the number of children should be agreed by the couple the conception was already reported by Farrokh-Eslamlou et al. (2014) in their study. Finally, it was found that $43.9 \%$ of women were willing to have children if the encouraging policies would well execute. This opinion was strengthened by Billari (2008)'s work but weakened by Mahmoudi et la. (2013) study.

Conclusively, as illustrated in the regression equation some elements such as living place, income rate, the number of children, providing facilities like free foodstuffs basket or considering retirement insurance for housewives are predictable to increase the childbearing decision-making. The data suggests that most of the women agree with social and financial policies that have been executed by the government and are willing to increase their number of children, but the government's encouraging proceedings in 4 provinces especially in Western Azarbayejan were just shown the reverse opinion where $80 \%$ of women shown their unwillingness to have more children even by governmental encouraging policies and proceedings (Erfani, 2013).

The research and ethics committee of Jahrom University of Medical Sciences has approved the study protocol and approval ID is Jums.REC.1393.026. All 
participants signed an informed consent prior to participating in the study.

Acknowledgements:

This research was partially supported by Jahrom University of Medical Sciences. I would like to thank Dr. Mehdi Abdoli for his motivation, and immense knowledge.

\begin{tabular}{llllllllr}
\multicolumn{8}{c}{ Table 1- Individual's characteristics } \\
\hline $\begin{array}{l}\text { Para- } \\
\text { Meter }\end{array}$ & Mean & \multicolumn{2}{l}{$\begin{array}{l}\text { 95\% Confidence SD } \\
\text { Interval for Mean } \\
\text { Lower }\end{array}$} & $\begin{array}{l}\text { Mpper } \\
\text { mum- Max-Range }\end{array}$ \\
\hline Age & 29 & 28 & 31 & 8.751 & 15 & 49 & 34 \\
Income & 391.19 & 300.24 & 482.14 & 780.47 & 0 & 7000 & 7000 \\
$\begin{array}{l}\text { Marriage } \\
\text { duration }\end{array}$ & 9.57 & 8.50 & 10.64 & 9.14 & .0 & 30 & 30 \\
$\begin{array}{l}\text { Total Son } \\
\text { Total }\end{array}$ & 5.6 & 4.7 & 6.6 & .81 & 0 & 4 & 4 \\
$\begin{array}{l}\text { Daughter } \\
\text { Spouse }\end{array}$ & 1285.5 & 4.5 & 6.7 & .93 & 0 & 5 & 5 \\
income & 1184.24 & 1387.32 & 870.77 & 0 & 5000 & 5000 \\
\hline
\end{tabular}

Table 2- The frequency of response to the degree of agreement about Incentive policies for childbearing

\begin{tabular}{|c|c|c|c|c|c|}
\hline & $\begin{array}{l}\text { tally } \\
\text { sagree } \\
\% \% \\
\end{array}$ & $\begin{array}{l}\text { Somewhat } \\
\text { disagree } \\
\mathrm{n} / \%\end{array}$ & $\begin{array}{l}\text { No } \\
\text { comme } \\
\mathrm{n} / \% \\
\end{array}$ & $\begin{array}{l}\text { agree } \\
\mathrm{n} / \%\end{array}$ & $\begin{array}{r}\text { Totally } \\
\text { agree } \\
\mathrm{n} / \% \\
\end{array}$ \\
\hline & & & & & 14 \\
\hline 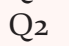 & 19 & 471 & $00 / 9$ & $264 / 39$ & $145 / 21.8 \%$ \\
\hline & & & & & \\
\hline 4 & $3 \%$ & & 69 & 208 & $233 / 32.1 \%$ \\
\hline 5 & $100 /$ & $186 /$ & $108 / 1$ & $150 /$ & $120 / 18.1 \%$ \\
\hline 6 & $34 / 5$ & $37 /$ & $55 / 8$. & 242 & $297 / 4$ \\
\hline $\mathrm{Q}_{7}$ & $102 / 1$ & $80 /$ & $131 / 1$ & $194 / 2$ & $158 / 2$ \\
\hline & $54 / 8$. & & & & \\
\hline & $92 / 1$ & $139 / 2$ & $193 / 2$ & $138 / 2$ & $101 / 15.2 \%$ \\
\hline & $108 /$ & & $171 / 2$ & $168 / 2$ & $118 / 17.8 \%$ \\
\hline 11 & $58 / 8$ & $75 /$ & $157 / 2$ & $172 / 25.9 \%$ & $201 / 30.3 \%$ \\
\hline 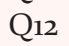 & & & $125 / 1$ & & \\
\hline$Q_{13}$ & $38 / 5$. & $37 / 5.6 \%$ & $104 / 15.6 \%$ & $202 / 30.4 \%$ & $284 / 42.7 \%$ \\
\hline
\end{tabular}

$\mathrm{Q}=$ question; $\mathrm{n}=$ total number; $\%=$ total percentage

\begin{tabular}{lll}
\hline \multicolumn{3}{l}{ Table3- The number of children, participants wish to have } \\
\hline Number of child & Frequency & Valid Percent \\
\hline 0 & 2 & 0.3 \\
1 & 24 & $3 \cdot 7$ \\
2 & 174 & 26.5 \\
3 & 325 & 49.5 \\
4 & 129 & 19.6 \\
5 & 1 & 0.2 \\
6 & 1 & 0.2 \\
7 & 1 & 0.2 \\
\hline
\end{tabular}

Table 4- Decision maker for childbearing in the family

\begin{tabular}{lll}
\hline Family member & Frequency & Valid Percent \\
\hline Husband & 67 & 10.2 \\
Wife & 66 & 10.1 \\
Both & 512 & 78.3 \\
Other & 9 & 1.4 \\
\hline
\end{tabular}

Table 5- Prediction of changing insight

\begin{tabular}{lllllllll}
\hline Variables & B & S.E. & Wald & df & Sig. & $\begin{array}{l}\text { Exp } \\
\text { (B) }\end{array}$ & \multicolumn{2}{l}{$\begin{array}{l}\text { 95.0\% C.I.for } \\
\text { EXP(B) } \\
\text { Lower Upper }\end{array}$} \\
\hline Residency & 0.848 & 0.371 & 5.225 & 1 & 0.022 & 2.336 & 1.129 & 4.834 \\
Child.no & -0.560 & 0.104 & 28.781 & 1 & 0.000 & .571 & 0.466 & 0.701 \\
q2 & 0.255 & 0.112 & 5.162 & 1 & 0.023 & 1.290 & 1.036 & 1.608 \\
q7 & 0.421 & 0.102 & 17.071 & 1 & 0.000 & 1.524 & 1.248 & 1.860 \\
q12 & 0.268 & 0.125 & 4.564 & 1 & 0.033 & 1.307 & 1.022 & 1.672 \\
q14 & 0.822 & 0.185 & 19.669 & 1 & 0.000 & 2.274 & 1.582 & 3.270 \\
Constant & -6.224 & 0.926 & 45.152 & 1 & 0.000 & 0.002 & & \\
\hline
\end{tabular}

\section{References:}

Abbasi-Shavazi, M.J. ( 200o): Convergence of fertility behaviors in Iran: Provincial fertility levels, patterns and trends: 19722000. Soc. Sci.J. (Persian), 18: 201-231.

Abbasi-Shavazi, M.J., \& McDonald, P. (2005): National and provincial-level fertility trends in Iran, 1972-200o, Working Papers in Demography, No. 94, Australian National University, Canberra.

Aghajanian, A. (1991): Population Change in Iran, 1966-86: A Stalled Demographic Transition? Popul. Dev. Rev.,17(4):703715 .

Andersson, G., Kreyenfeld, M. \& Mika, T. (2014): Welfare state context, female labour-market attachment and childbearing in Germany and Denmark, J.Popul. Res., 31( 4), 287-316.

Billari, F.C. (2008): Lowest-Low Fertility in Europe: Exploring the Causes and Finding Some Surprises. Japan. J. Popul.,6(1):1-17.

Eberstadt, N. \& Shah, A. (2013): On Fertility Decline in the Muslim World: A Veritable Sea-Change, Still Curiously Unnoticed, in H. Groth \& A. Sousa-Poza (eds.), Population Dynamics in Muslim Countries, Springer-Verlag Berlin Heidelberg .

Erfani, A. (2013): A Reversal in the Population Policy of Iran: Do Curbin Family Planning Programs Raise Low Fertility? IUSSP, Busan, Korea p. 1-4.

Farrokh-Eslamlou, H.R. , Vahabzadeh, Z., Moeini, S.R., Tabrizi, M.F.( 2013): Pre-Marriage Couples Fertility Attitude Following Recent Childbearing Persuasive Policies In Iran. L.Urmia Nurs. Midwifery Facul., 11(10): 836-846. [Persian]

Kalantari, S., Abaszadeh, M., Amin-Mozafari, F., Rakei-Bonab, N. (2010): Socialogic evaluation of aff inity to child bearing and some related factors: Married adolescents of Tabriz district case study.Appl. Soc.,37(1):83-104. (Persian)

Kariman, N., Simbar, M., Ahmadi, F. \& Vedadhir, A.A. (2014): Concerns about One's Own Future or Securing Child's Future: Paradox of Childbearing Decision Making. Health, 6, 1019-1029.

Mahmoudi, M., Kazemiour, S., Ahrari, M. \& Nikoonesbati, A. (2013): Predicting Iran's Population Growth Considering Socioeconomic Variables: A Multidisciplinary Approach. Plann. Budg. Quart., 17:97-126.

Mansourian, M.K., Khoshnevis, A. (2006): Married women's attitude and sexual preferences about reproductive practice: Tehran case study. Hum soc sci J., 24(2):129-46. (Persian)

Shadi-Talab, J. (2001): Iranian women: Rising expectations. Paper presented at MESA, 27-28.

U.N. Stats.(2013): Iran's Slow Population Growth, United States Institute of Peace, The Iran Primer, 1-2. 